\title{
Modelo de Gestión de Costos Ocultos para la Mejora Continua de los Recursos Humanos
}

\author{
Hidden Cost Management Model \\ for the Continuous Improvement of Human Resources \\ Ana María NEGRÓN-GONZÁLEZ1 (iD, Germán GEMAR-CASTILLO'D y Marcia Esther NODA-HERNÁNDEZ² iD \\ 1 Universidad de Málaga, Facultad de Ciencias Económicas y Empresariales. Málaga, España. \\ 2 Ministerio de Educación Superior de Cuba, Junta de Acreditación Nacional. La Habana, Cuba.

\begin{abstract}
Resumen
Una adecuada identificación y cuantificación de costos ocultos de la gestión de recursos humanos contribuye a aumentar la efectividad en la gestión empresarial. Actualmente, en muchas empresas no se reflejan los costos ocultos en sus balances económicos, ni en sus registros financieros.

El objetivo es analizar los resultados de la aplicación de un modelo de gestión de costos ocultos para la mejora continua de los recursos humanos en el sector hotelero. Se ha tomado en cuenta en todo momento las expectativas y necesidades de los clientes y se ha integrado los elementos sociales y económicos de la gestión socioeconómica para contribuir al desarrollo de la calidad del servicio en el sector hotelero.

Se han utilizado diferentes técnicas y herramientas, tales como la lista de chequeo, el cuadro de mando integral, el modelo SERVQUAL y un estudio empírico del cual se hizo un análisis factorial como método de reducción de datos, para obtener las correlaciones entre más de las 57 variables observables. Este estudio permitirá alcanzar niveles superiores en el desempeño laboral, además de un incremento en la productividad del trabajo y calidad del servicio.
\end{abstract}

Palabras clave

Gestión socioeconómica, gestión de recursos humanos, costos ocultos.

\section{Abstract}

Proper identification and quantification of hidden costs of human resource management contributes to increasing business management effectiveness. Currently, in many companies the hidden costs are not reflected in their financial balance sheets, or in their financial records.

The objective is to analyze the results of the application of a hidden cost management model for the continuous improvement of human resources in the hotel sector. The expectations and needs of customers have been taken into account at all times and the social and economic elements of socio-economic management have been integrated to contribute to the development of service quality in the hotel sector.

Different techniques and tools have been used, such as the checklist, the balanced scorecard, the SERVQUAL model and an empirical study of which a factor analysis was carried out as a data reduction method, to obtain the correlations between more of the 57 observable variables. This study will allow reaching higher levels in job performance, in addition to an increase in work productivity and quality of service.

\section{Keywords}

Socio-economic management, human resource management, hidden costs.

Códigos de clasificación JEL: M12, M21, M50. 


\section{Introducción}

Ante la demanda creciente de información del funcionamiento y de los efectos de la gestión empresarial, las empresas se ven obligadas a incrementar la transparencia, por lo que adoptan estrategias de comunicación que demuestren que no solo la empresa, sino que sus proveedores y subcontratistas son respetuosos con su personal. Para contribuir a ello, se gestionan los recursos humanos a través de la aplicación de políticas y de diferentes enfoques; y un enfoque poco estudiado es el económico, lo mismo que su presencia en el perfeccionamiento empresarial.

En los modelos de gestión de recursos humanos $(\mathrm{GRH})$ se puede apreciar diferencias dadas por su estructura e interacciones entre los elementos que los componen, además distinguirse por la forma de abordar el problema social y su repercusión económica (Gemar-Castillo et al., 2019).

Por ejemplo, el modelo de Harper y Lynch (1992) está orientado fundamentalmente hacia el cumplimiento de la estrategia de la organización y optimización de los recursos humanos. Para ello en el modelo se plantea las auditorías, las cuales son fundamentales para controlar los recursos humanos. Werther y Davis (2008), por el contrario, no incorporan a su modelo el diagnóstico de los recursos humanos, ni su vinculación con los costos, aunque sí consideran necesario evaluar los principales procesos de la GRH. Otros autores como Chiavenato (2017) y Werther y Davis (2008) proponen un modelo de diagnóstico de recursos humanos donde el balance social es un elemento primordial en el que se centran los resultados finales deseables, así como su control, a través de la retroalimentación. Beer et al. (1985) y Cuesta Santos y Valencia Rodríguez (2014) se centran en los procesos y los declaran como elementos fundamentales en la GRH, siempre teniendo en cuenta las consecuen- cias a largo plazo que tienen para la organización, estos últimos (Cuesta Santos \& Valencia Rodríguez, 2014), además, tienen en cuenta a la sociedad en los grupos de interés y desarrollan la retroalimentación del sistema a través de los resultados de la auditoría y control de gestión, punto de partida en la evaluación de su impacto económico (Cuesta Santos \& Valencia Rodíguez, 2018).

Los modelos de recursos humanos han ido evolucionando a una gestión por competencias. Alles (2006) considera que la gestión por competencia agrega valor a la estrategia de negocio. Pereda y Berrocal (2018), que proponen un enfoque de competencias, plantean la necesidad de un programa de evaluación del rendimiento y del potencial.

El modelo de Morales Cartaya (2009) parte de nueve módulos constituidos por los procesos fundamentales del capital humano, donde las competencias son el centro de la gestión para las organizaciones, al igual que la planificación estratégica de los recursos humanos a corto, mediano y largo plazo. El perfil de competencias se consideran el primer eslabón que expresa la integración de la gestión del capital humano con la estrategia empresarial. Entre los procesos para reducir costos constan la capacitación y desarrollo, estimulación moral y material, la organización del trabajo y la seguridad y salud en el trabajo (Morales Cartaya, 2009).

Este modelo cuenta con un sistema de normas aprobadas por la Oficina Nacional de Normalización para el Sistema de Gestión Integrada de Capital Humano: NC 3000: 2007, NC 3001: 2007 y NC 3002: 2007, las cuales constituyen una experiencia cubana muy particular y única en el marco de las normas de gestión organizacional reconocidas por los órganos internacionales de normalización (ONN, 2007a, 2007b, 2007c). En este modelo no aparece declarada su incidencia en la economía de la empresa, ni cómo pueden aportar a la disminución de sus costos. Uno de los 
elementos que pueden influir directamente en esto son las variables sociales, las cuales están muy relacionadas con el capital humano (Negrón-González et al., 2020).

En el modelo de la norma cubana, el autocontrol es uno de los procesos que está dirigido a una actividad sistemática de control que ejecuta la organización, para medir el impacto en el logro de los objetivos y la estrategia a partir de evaluar, en la práctica, la eficacia de los procesos de la Gestión Integrada de Capital Humano (GICH) (ONN, 2007a, 2007b, 2007c). La aplicación de este proceso puede proporcionar una visión global del desempeño de la organización y del grado de madurez del sistema de GRH. Asimismo, puede ayudar a identificar los procesos y las actividades que precisan mejoras, y a determinar las prioridades.

Uno de los elementos esenciales en el control de los recursos humanos es el Cuadro de Mando Integral que complementa los indicadores financieros de la actuación pasada con las medidas de los inductores de la actuación futura. Los indicadores contemplan la actuación de la organización desde cuatro perspectivas: la financiera, la del cliente, la del proceso interno y la de formación y crecimiento (Kaplan \& Norton, 2014).

La perspectiva de formación y crecimiento está asociada a los recursos humanos. A través de ella se identifica la infraestructura que la empresa debe construir para impulsar una mejora y crecimiento a largo plazo. Asimismo, las perspectivas del cliente y de los procesos internos sirven para identificar los factores más críticos para el éxito actual y futuro. Por otra parte, la intensa competencia global exige que las empresas mejoren continuamente sus capacidades para entregar valor a sus clientes y accionistas (Kaplan \& Norton, 2014). Precisamente, la formación y crecimiento de una organización impulsan la mejora de estas capacidades, y proceden de tres fuentes principales: las personas, los sistemas y los procedimientos (Gemar-Castillo et al., 2019).

Uno de los enfoques económicos tratados, con énfasis en los recursos humanos, es el socioeconómico. El Instituto de SocioEconomía de las Empresas y de las Organizaciones (ISEOR) lleva a cabo estas investigaciones. Entre las tareas del ISEOR figura constatar que las herramientas usuales de análisis de gestión no permiten registrar los desperdicios de energía y de recursos que están latentes en la empresa u organización, es decir, los costos-desempeños ocultos (Parra-Acosta \& Peña-González, 2014).

De acuerdo con la teoría socioeconómica de las organizaciones, la organización es un sistema humano que se encuentra inmerso en un sistema biológico más amplio, por lo que su desempeño económico no puede separarse del social; pues el primero sin el segundo redunda a la larga en costos ocultos, producto de los disfuncionamientos que se manifiestan cuando estos dos aparecen disociados (Savall \& Zardet, 2017). Los disfuncionamientos son agrupados en seis familias: condiciones de trabajo, organización del trabajo, comunicación, coordinación, formación integrada.

En Cuba, desde 1998, se lleva a cabo en el sistema empresarial un proceso de cambio impulsado por el $V$ Congreso del PCC. Este modelo tiene como objetivo lograr la competitividad empresarial.

En resumen, la situación es la siguiente: El $87 \%$ de los encuestados de más de 40 hoteles de La Habana dijeron que no identifican los costos ocultos asociados en todos los procesos de $\mathrm{GRH}$; Existen disfuncionamientos asociados a los procesos de GRH que no son identificados y que no se relacionan con el incumplimiento de las expectativas y necesidades de los clientes; Existen costos que se pueden cuantificar o prever su existencia para así controlar su comportamiento, sin embargo, actualmente esto no se aplica. 
El objetivo fundamental propuesto para esta investigación es analizar los resultados de la aplicación de un modelo de gestión de costos ocultos para la mejora continua de los recursos humanos en el sector hotelero.

\section{Revisión de la literatura}

Las empresas cuentan con recursos tangibles e intangibles, los cuales son esenciales para su funcionamiento y eficiencia (Abad Alfonso et al., 2016; Brínez et al., 2016; Mesa et al., 2009). Estos recursos se agrupan en diferentes tipos: físicos, humanos, financieros e intelectuales (Herrera Ricardo et al., 2020). En el caso del desarrollo de los recursos asociados con el desarrollo directo de los recursos humanos, existen las interacciones entre individuos, organizaciones y fuerzas nacionales en todos los países (Alagaraja \& Githens, 2016), mientras que la administración de los recursos humanos se está enfocando con más frecuencia en las políticas, el personal, las TIC relacionadas con los recursos humanos y la compensación (Blackman et al., 2016).

Después de analizar en los modelos clásicos de GRH, la presencia del enfoque económico y su contribución al desarrollo de la empresa, así como su incidencia directa a la satisfacción del cliente, se resumen los principales resultados obtenidos en la tabla 1.

Tabla l. Análisis de los Modelos de Gestión de Recursos Humanos

\begin{tabular}{|c|c|c|}
\hline $\begin{array}{l}\text { Modelos de Gestión } \\
\text { de Recursos Humanos }\end{array}$ & Enfoque económico & Satisfacción del cliente \\
\hline Beer et al. (1985) & $\begin{array}{l}\text { Enfocados en los procesos. Desarrollan la retroali- } \\
\text { mentación del sistema a través de los resultados } \\
\text { de la auditoría y control de gestión. }\end{array}$ & $\begin{array}{l}\text { Clientes internos y externos } \\
\text { reunidos en grupo de interés. }\end{array}$ \\
\hline Harper y Lynch (1992) & $\begin{array}{l}\text { Las auditorías son elementos importantes para } \\
\text { controlar los recursos humanos. }\end{array}$ & Enfoque funcional \\
\hline Quintanilla (1991) & $\begin{array}{l}\text { Formula la existencia de cuatro funciones entre } \\
\text { las cuales plantea el subsistema de integración y } \\
\text { socialización. }\end{array}$ & Cliente interno \\
\hline Chiavenato (2017) & $\begin{array}{l}\text { Plantea un modelo de diagnóstico y control de re- } \\
\text { cursos humanos, a través de la retroalimentación. }\end{array}$ & Cliente interno y externo \\
\hline Werther y Davis (2008) & $\begin{array}{l}\text { Proponen la evaluación de los principales procesos } \\
\text { de la GRH. }\end{array}$ & Cliente interno \\
\hline Alles (2006) & $\begin{array}{l}\text { Considera que la gestión por competencia agrega } \\
\text { valor a la estrategia de negocio. }\end{array}$ & Cliente interno y externo \\
\hline Pereda y Berrocal (2018) & $\begin{array}{l}\text { Enfocados en las competencias, plantean la nece- } \\
\text { sidad de un programa de evaluación del rendimien- } \\
\text { to y evaluación del potencial. }\end{array}$ & Cliente interno y externo \\
\hline Cuesta Santos (2012) & $\begin{array}{l}\text { Contempla un diagnóstico, parte de la orientación } \\
\text { estratégica y la retroalimentación del sistema a } \\
\text { través de los resultados de la auditoría y control de } \\
\text { gestión. }\end{array}$ & $\begin{array}{l}\text { Clientes internos y externos } \\
\text { reunidos en grupo de interés. }\end{array}$ \\
\hline
\end{tabular}

En los modelos analizados no aparecen las relaciones directas de los procesos de recursos humanos con la economía de la empresa ni cómo pueden ayudar a disminuir sus costos. Uno de los elementos que pueden hacer caer la balanza en la optimización 
del coste de personal son las variables sociales, especialmente en la administración estratégica de recursos humanos que, en el último cuarto de siglo, se ha observado como una tendencia mundial la consideración y relevancia de estas variables en la práctica ejecutiva y donde es necesario reinventar los fundamentos éticos de la gestión de personas (Bermúdez, 2014).

Es importante que los directivos de recursos humanos incorporen métodos analíticos para considerar el propósito de estos esfuerzos (King, 2016). Por ejemplo, en unos estudios llevados a cabo en Rumania se destacó al recurso humano como un factor determinante en el desarrollo sostenible, a partir del impacto del bajo nivel de competencias de los trabajadores y el alto costo que implica la desprofesionalización de los recursos humanos, debido al exceso de rotación de personal, entre otras razones (Chitescu \& Lixandru, 2016).

Asimismo, por medio de un estudio realizado en Nigeria sobre la influencia del costo de los recursos humanos en la productividad del trabajo se demostró cómo el desarrollo de estos puede tener un impacto positivo, en la medida en que la empresa tenga éxito al desarrollar las habilidades y conocimientos de sus empleados (competencias), además de que el costo de desarrollo (capacitación) es alto y significativamente relacionado con el desempeño de las empresas (Bassey \& Tapang, 2012).

En suma, se considera que esta fuerza de trabajo posee un importante componente humanista, al implicarse socialmente la persona o pertenecer a una comunidad, lo mismo que el componente económico al asociarse al aumento de la productividad del trabajo o al alto desempeño (Montoya Monsalve \& Montoya Naranjo, 2012; Peralta Gómez et al., 2007). Además, se reconoce que los empleados se vuelven más responsables y cuidadosos en el trabajo cuando se sienten apreciados (Blaga \& Jozsef, 2014).
El control de los recursos humanos es esencial para contribuir aumentar la productividad del trabajo, y una herramienta fundamental para este fin es el cuadro de mandos integral. En este cuadro se complementan los indicadores financieros de la actuación pasada con las medidas de los inductores de la actuación futura a través de cuatro perspectivas: financiera, cliente, proceso interno y formación y crecimiento (Kaplan \& Norton, 2014). La perspectiva de formación y crecimiento está asociada a los recursos humanos y sirve para identificar la infraestructura que la empresa debe construir para crear una mejora y crecimiento a largo plazo. Las perspectivas del cliente y del proceso interno sirven para identificar los factores más críticos para el éxito actual y futuro. También se utiliza para evidenciar los nexos causales o hipótesis entre los diferentes objetivos estratégicos planteados (Cuesta Santos, 2016; Cuesta Santos \& Valencia Rodríguez, 2014).

De igual manera, se plantea que los costos deberían agrupar el desarrollo de los recursos humanos, y que deberían incluir una serie de honorarios pagados en la contratación de personal, selección, disposición, utilización, orientación y en el trabajo de formación (Jinhua, 2016); todo ello encaminado a buscar el efecto de la reducción de costos y la estrategia empresarial en las prácticas de subcontratación de los recursos humanos (Mansor et al., 2017). La perspectiva económica es fundamental para el éxito de la empresa, por lo que lograr establecer unos vínculos claros entre la gestión de recursos humanos y los resultados económicos de la organización es un aspecto esencial en esta investigación.

Uno de los enfoques económicos tratados por la investigación en recursos humanos es el socioeconómico. Estas investigaciones son realizadas por el Instituto de Socio-Economía las Empresas y de las Organizaciones (ISEOR), Centro de investigación asociado a la Universidad Jean Moulin Lyon y 
a la Escuela de Management de Lyon, Francia, donde entre sus tareas se encuentra constatar que las herramientas usuales de análisis de gestión no permiten registrar los desperdicios de energía y de recursos que están latentes en la empresa u organización: se trata de los costos-desempeños ocultos (Savall \& Zardet, 2019).

Las disfunciones, según Savall y Zardet (2016), se agrupan en seis familias: condiciones de trabajo, organización del trabajo, comunicación, coordinación, concertación, gestión del tiempo, formación integrada y puesta en marcha estratégica (Parra-Acosta \& Peña-González, 2014; Savall \& Zardet, 2016). Estos campos están limitados únicamente a los procesos de organización del trabajo, comunicación y formación de la GRH. Esta es una de las líneas de investigación en esta propuesta de tesis doctoral, la cual abarca todos los procesos de la GRH entre los que se encuentra el flujo de recursos humanos, administración estratégica de los recursos humanos, comunicación institucional, organización del trabajo, evaluación del desempeño, selección e integración, capacitación y desarrollo, autocontrol, seguridad y salud en el trabajo, y estimulación moral y material (Cuesta Santos, 2015).

Los costos ocultos asociados a las disfunciones existentes son definidos como la diferencia entre el funcionamiento deseado y el funcionamiento real, los cuales no son identificados en los sistemas de información de la empresa - presupuesto, contabilidad general y analítica, tablero de conducción(Savall, 2011). Este método muestra cómo algunas actividades hechas en la organización tienen un impacto financiero en ella, aunque muchas veces pasen inadvertidas en los informes financieros tradicionales, ya que pueden ser camufladas en otras partidas que no reflejan plenamente su realidad, o puede que no estén contabilizadas porque corresponden a disminuciones en los flujos de caja.
Aunque estos procesos no sean visibles en los sistemas tradicionales contables, sí repercuten en el comportamiento de la empresa (Parra-Acosta \& Peña-González, 2014).

Bampoky (2012) propone indicadores para agrupar los costos ocultos, tales como: absentismo, cantidad de accidentes de trabajo, índice de rotación del personal, no calidad y falta de productividad. La evaluación sistemática de estos indicadores permitirá mejorar los procesos internos de la empresa. Hay que tener en cuenta que no solo es importante conocer el costo total de un producto o servicio, sino que también es necesario conocer otros elementos que repercuten negativamente en la cadena de valor, pero que se escapan de los métodos y herramientas de control de gestión tradicionales (Bampoky, 2012).

La efectividad de la GRH está asociada con la inversión en recursos humanos y con la minimización estratégica de costos (Vardarlıer, 2016). Así también, hay quienes han investigado acerca del uso de tecnología para facilitar los procesos de recursos humanos, lo que típicamente mejora la eficiencia y disminuye los costos asociados con las transacciones de recursos humanos (Stone \& Deadrick, 2015).

\section{Materiales y métodos}

Se plantea un modelo de gestión de costos ocultos para la mejora continua de los recursos humanos tomando en cuenta las siguientes bases teórico-metodológicas del procedimiento:

- Enfoque en un sistema: se considera que la estructura de la empresa es un sistema complejo de relaciones que trabaja para responder a las demandas del entorno a partir de la integración de sus subsistemas con la estrategia de la empresa, y así obtener un 
resultado de alto impacto económico y social (Chiavenato, 2017).

- Enfoque a procesos: el diseño y modelación de los procesos a partir del modelo extendido de GRH (García \& Fleitas, 2013), permite revelar la interrelación de los procesos, desde el sistema de trabajo, del que se extrae el flujo de los recursos humanos y en donde se priorizan las relaciones entre los procesos que atraviesan la organización para agregar valor en el cumplimiento de la satisfacción de las necesidades de la sociedad, los clientes y otras partes interesadas.

- El modelo de gestión del capital humano de Cuesta Santos (2012), modificado para el diagnóstico y los procedimientos que se tienen en cuenta para el diseño de los procesos.

- El modelo de Morales Cartaya (2009) y las NC 3000, 3001 y 3002 (ONN, 2007a, 2007b, 2007c), modelo de $\mathrm{GRH}$, donde se utilizaron los nueve procesos para la GCH y partiendo de ellos se encontraron los disfuncionamientos que existían en las empresas seleccionadas.

- El modelo de gestión socio-económica desarrollado por la ISEOR que define conjuntamente objetivos económicos y objetivos sociales, los cuales permiten identificar los disfuncionamientos existentes en los recursos humanos y cuantificar sus costos (Savall \& Zardet, 2019).

El modelo para la identificación y control de los costos ocultos de los recursos humanos, teniendo en cuenta las expectativas y necesidades de los clientes, que integra los elementos sociales y económicos, y que contribuye al desarrollo de la calidad del servicio en hoteles habaneros, se muestra en la figura 1.

Figura 1. Modelo de gestión de costos ocultos para la mejora continua de los recursos humanos

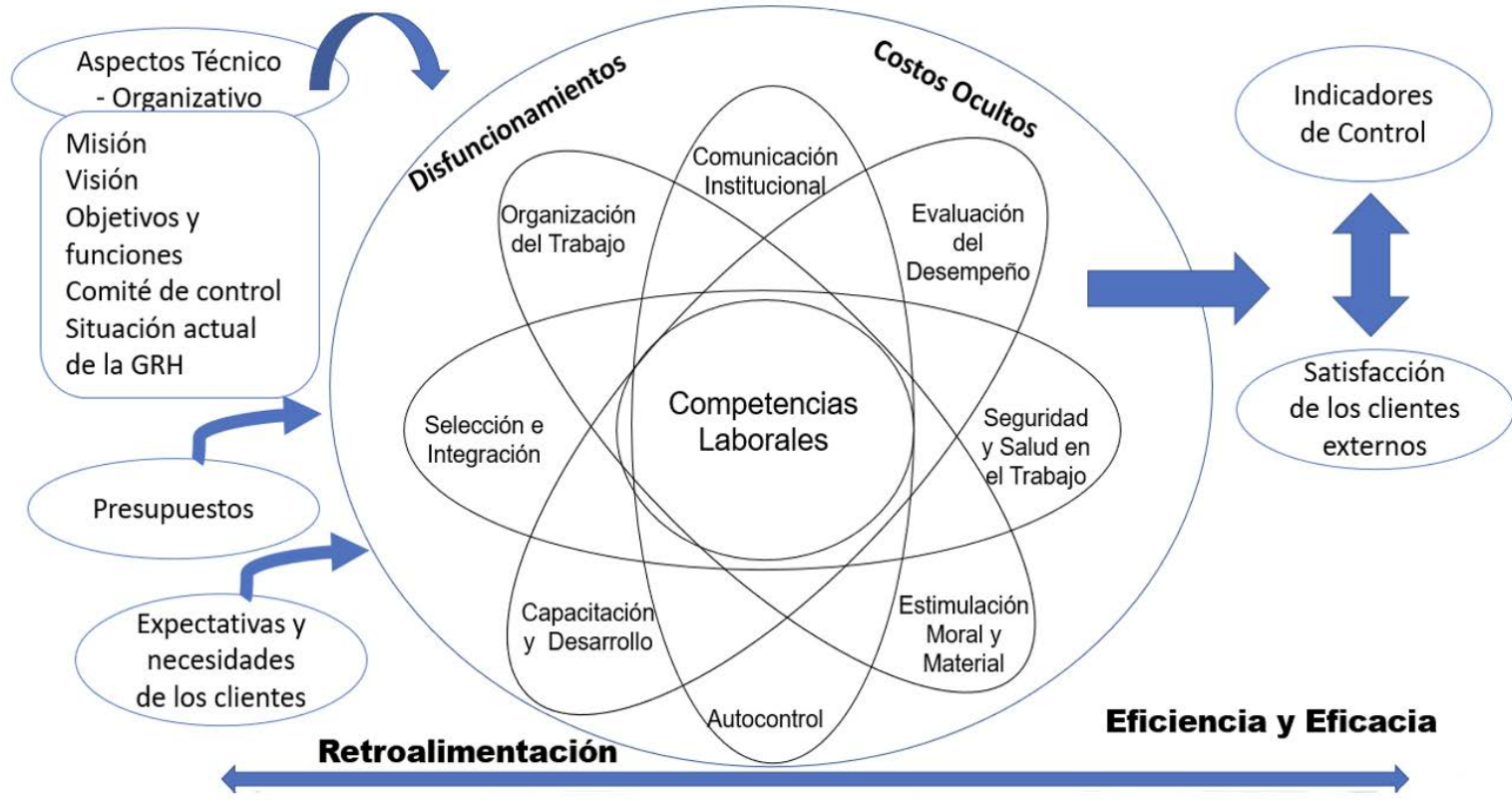

El modelo que se propone en esta investigación pretende abarcar todos los procesos de recursos humanos y utilizar los estudios técnicos de cada proceso para identificar las disfunciones, por lo que se aplicó una encuesta, debidamente aprobada y testada, a los clientes 
de los hoteles seleccionados, con base en el modelo SERVQUAL. El modelo SERVQUAL permite identificar variables relacionadas directamente con la GRH, tales como: habilidad e interés por enfrentar los problemas de los clientes, empleados que inspiran confianza, apariencia del personal, entre otros. Este modelo define la calidad del servicio como la diferencia entre las percepciones reales de los clientes respecto del servicio y las expectativas formadas previamente sobre este. De esa forma, un cliente valora como negativa o positiva la calidad de un servicio teniendo en cuenta si las percepciones que ha obtenido han sido inferiores o superiores a las expectativas que tenía (Jasinskas et al., 2016; Matsumoto, 2014)

A pesar de que esta metodología es una de las más usadas en el mundo, no está exenta de críticas. Por ejemplo, desde un punto de vista teórico, la operacionalización de las percepciones y expectativas ha sido criticada porque la evidencia de que los consumidores evalúen la calidad de servicio en función de la brecha existente entre percepciones y expectativas es escasa. Además, se suele centrar la atención en el proceso de entrega del servicio más que en el resultado de la provisión de este; es decir, en el encuentro del proveedor con el consumidor.

\section{Análisis y resultados}

Se muestran los resultados de la aplicación de este modelo en hotel habanero donde se reunió un equipo de trabajo. El equipo se formó con aquellos trabajadores que poseen un mayor conocimiento y experiencia en los costos ocultos de recursos humanos. El equipo estuvo integrado por un grupo de expertos que son los jefes de los departamentos de la Dirección de Desarrollo Humano y otros especialistas que tienen experiencia en esta actividad. En total se seleccionó a nueve personas.

Para la capacitación del equipo de trabajo, se hizo un diagnóstico de las nece- sidades de conocimientos a través de la encuesta de competencias de expertos que se aplicó a las nueve personas seleccionadas. De este grupo se escogió a seis expertos en el tema: siete están categorizados como expertos altos, por tener un coeficiente de competencia superior a $0.8[0.8 \leq \mathrm{K} \leq 1]$, y dos fueron clasificados como medio $[0.5 \leq \mathrm{K}$ $<0.8]$. El coeficiente de competencia promedio de los expertos seleccionados es de 0.87.

Posteriormente, partiendo de un análisis documental apoyado en más de 20 entrevistas, se puede apreciar que: los procesos de capital humano no están diseñados o documentados, se cuenta con las regulaciones vigentes del Ministerio de Trabajo y Seguridad Social, se realiza un estudio del actual informe económico del centro, así como la desagregación del presupuesto y los planes de ingresos, y en el análisis de estos se utilizan indicadores como productividad, salario medio, relación salario medio/productividad, gastos, ingresos, rentabilidad, entre otros.

El modelo SERVQUAL se aplicó a más de 75 clientes para conocer su nivel de satisfacción. De este modelo se obtuvo la evaluación de calidad de los servicios ofrecidos por el hotel, según las expectativas y percepciones del cliente como se muestra en la figura 2. Además, se conoció cuáles ítems relacionados con la GCP influyen directa y negativamente en la satisfacción del cliente externo.

Al obtener la valoración global de la calidad del servicio y la de las cinco dimensiones analizadas, se concluye que existe déficit de calidad en los siguientes ítems:

- Ítem 1: Los elementos materiales (folletos, estados de cuenta y similares) son visualmente atractivos (elementos tangibles).

- Ítem 5: Cuando la empresa de servicios promete hacer algo en cierto tiempo, lo cumple (fiabilidad).

- Ítem 17: Los empleados tienen conocimientos suficientes para respon- 
der a las preguntas de los clientes (seguridad).

De los ítems evaluados como deficientes por los clientes, se considera que tienen relación directa con la GCH los correspondientes a:

1. "Cuando la empresa de servicios promete hacer algo en cierto tiempo, lo hace".

2. "Los empleados tienen conocimientos suficientes para responder a las preguntas de los clientes"
3. "La empresa de servicios comprende las necesidades específicas de sus clientes", específicamente con el proceso referido a competencias laborales, ya que existen deficiencias en la capacitación y evaluación del desempeño del personal del hotel, así como en la organización del trabajo, lo que repercute directamente en la calidad del servicio y por ende en la satisfacción del cliente.

Figura 2. Resultados del Modelo SERVQUAL en el hotel

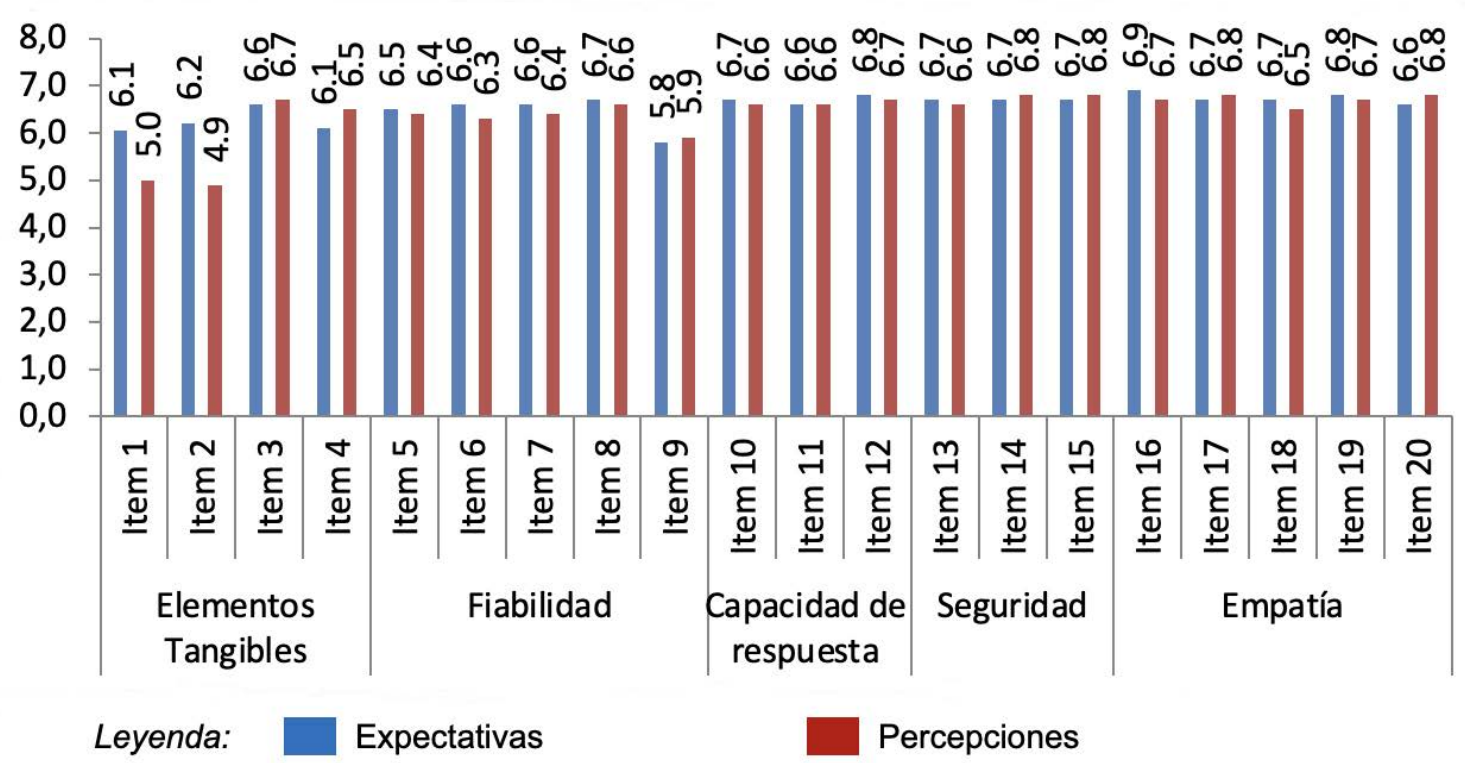

Los gastos registrados del capital humano en los estados financieros de la empresa solo corresponden a los procesos de seguridad y salud del trabajo, capacitación, comunicación y estimulación moral y material.

Una vez terminado el análisis en el hotel, se hizo un diagnóstico de los procesos que integran el Sistema de Gestión de Recursos Humanos y se identifican los disfuncionamientos existentes. En el diagnóstico

de los procesos de la GRH se obtuvo como principales resultados:

Se aplica al grupo de expertos del equipo de trabajo seleccionado la lista de chequeo para analizar el cumplimiento de los requisitos que establece la NC 3001:2007 vinculados a cada proceso. El proceso de competencias laborales presenta el cumplimiento más bajo, con un $6.7 \%$, como se muestra en la figura 3. 
Figura 3. Cumplimiento de los requisitos de la NC 3001:2007

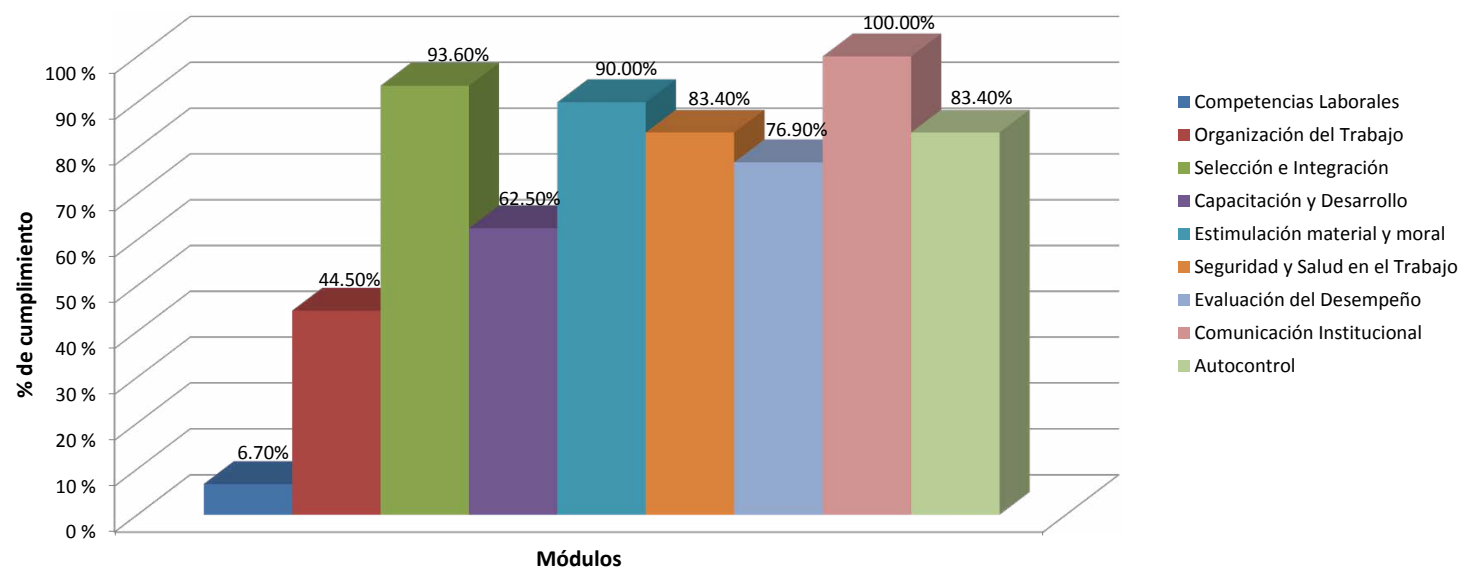

Partiendo de las deficiencias detectadas en los procesos que conforman la GRH, la información obtenida de la investigación documental y la aplicación de una nueva lista de chequeo, diseñada para relacionar la existencia de disfuncionamientos a partir del incumplimiento de los requisitos de la NC 3002: 2007 con cada proceso de recursos humanos, se obtuvieron los resultados que se muestran en la figura 4.

Figura 4. Disfuncionamientos asociados al incumplimiento de los requisitos de la NC: 3002: 2007

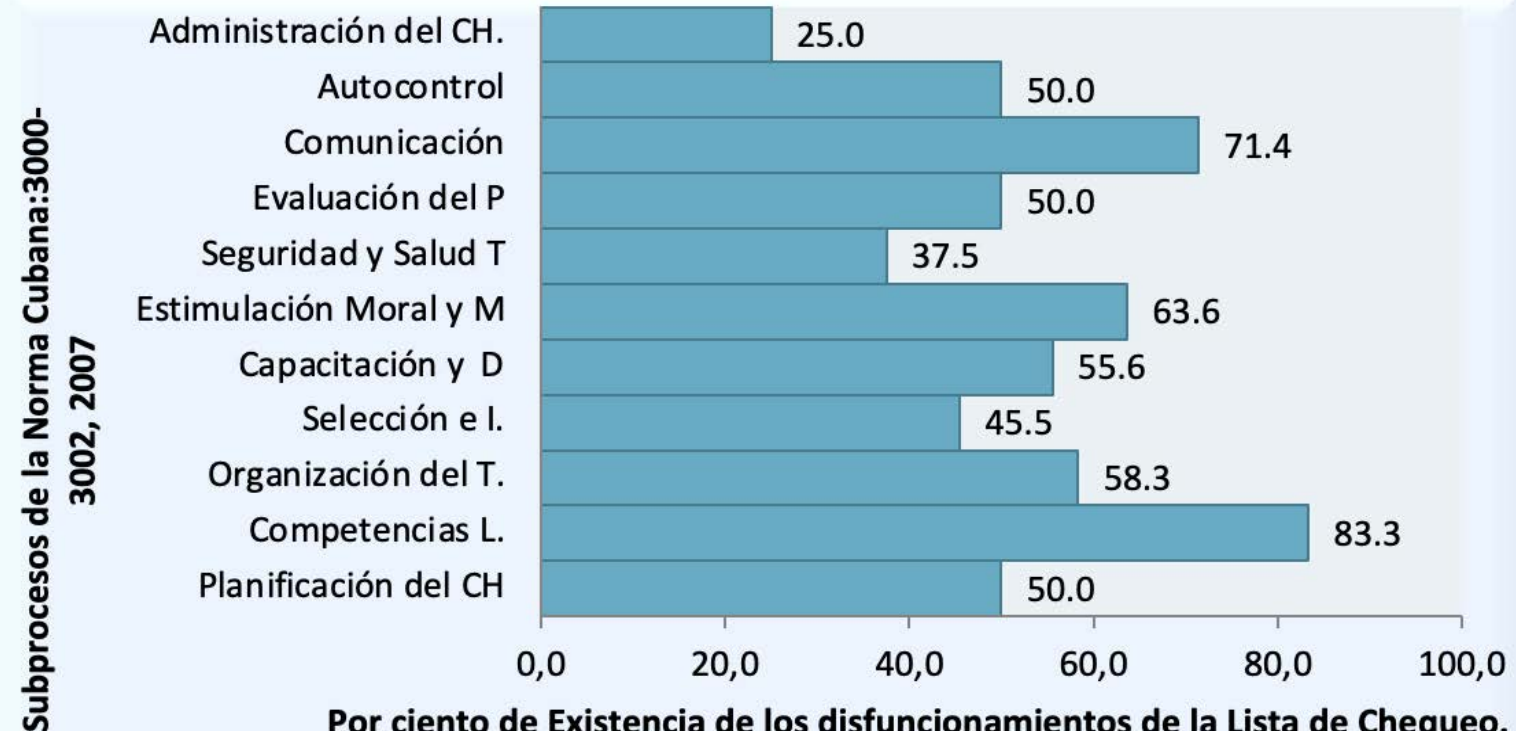

Los procesos que presentan mayores disfuncionamientos son las competencias laborales con un 83.3 \% y la comunicación con un $71.4 \%$. Los principales disfuncionamientos encontrados son los siguientes: existencia de trabajadores no idóneos para ocupar su cargo, fluctuación laboral, desmotivación, tiempos improductivos por parte de los trabajadores, existencia de trabajadores cuya evaluación del desempeño no se corresponde con su desempeño real (tabla 2). 
Tabla 2. Disfuncionamientos y costos ocultos

\begin{tabular}{|l|l|l|}
\hline \multicolumn{1}{|c|}{ Disfuncionamientos } & \multicolumn{1}{c|}{ Proceso } & \multicolumn{1}{c|}{ Costo oculto } \\
\hline Existencia de trabajadores no idóneos para ocupar el cargo & Selección del personal & Riesgo \\
\hline Tiempos improductivos por parte de los trabajadores & Organización del trabajo & No producción \\
\hline Deficiente gestión del tiempo & Formación & Sobretiempos \\
\hline $\begin{array}{l}\text { Existencia de trabajadores cuya evaluación del desempeño } \\
\text { no se corresponde con su desempeño real }\end{array}$ & Evaluación del desempeño & Riesgo \\
\hline Fluctuación laboral & Selección e integración & Riesgo \\
\hline
\end{tabular}

Para la cuantificación se tomó una muestra de los trabajadores que pertenecen al proceso de alojamiento. Se seleccionó este proceso porque le aporta mayor cantidad de ingresos al hotel. Se calcularon los costos ocultos asociados a los disfuncionamientos elementales identificados a través del método de costos $A B C$, donde se determinan por cada disfuncionamiento los elementos que lo componen y sus costos.

Tabla 3. Cuantificación de costos ocultos

\begin{tabular}{|c|c|c|c|c|}
\hline Subprocesos & $\begin{array}{l}\text { Disfuncionamien- } \\
\text { tos elementales }\end{array}$ & $\begin{array}{l}\text { Causas } \\
\text { invocadas }\end{array}$ & $\begin{array}{l}\text { Impacto sobre } \\
\text { el desempeño } \\
\text { económico }\end{array}$ & $\begin{array}{l}\text { Costos de los } \\
\text { disfuncionamientos } \\
\text { (costos ocultos) }\end{array}$ \\
\hline $\begin{array}{l}\text { Organización } \\
\text { del trabajo }\end{array}$ & $\begin{array}{l}\text { Tiempos improducti- } \\
\text { vos por parte de los } \\
\text { trabajadores } \\
(53.1 \%)\end{array}$ & $\begin{array}{l}\text { En ocasiones, } \\
\text { los trabaja- } \\
\text { dores están } \\
\text { motivados }\end{array}$ & $\begin{array}{l}\text { Afectaciones a } \\
\text { las cuotas de } \\
\text { producción }\end{array}$ & $\begin{array}{l}\text { Ausentismo: Por ausencias in- } \\
\text { justificadas: } 12 \text { días/año por } \\
\text { parte de } 1 \text { trabajador ( } 12 \text { días / } \\
\text { año*8 h/día * } 15 \text { USD/h =1440 } \\
\text { USD/año). } \\
\text { Impuntualidades por entrar al } \\
\text { hotel después de las } 8 \text { h35 o } \\
\text { salir antes de las 17h00: (171 h/ } \\
\text { año * } 87 \text { trabajadores *15 USD/h } \\
=223155 \text { USD/año. }\end{array}$ \\
\hline \multicolumn{5}{|c|}{\begin{tabular}{l|l} 
Total = 23366.7 USD/año
\end{tabular}} \\
\hline $\begin{array}{l}\text { Evaluación del } \\
\text { desempeño }\end{array}$ & $\begin{array}{l}\text { Existencia de traba- } \\
\text { jadores cuya evalua- } \\
\text { ción del desempeño } \\
\text { no corresponde con } \\
\text { su desempeño real } \\
(3.2 \%) \text {. }\end{array}$ & $\begin{array}{l}\text { En la evaluación } \\
\text { del desempeño } \\
\text { no se tienen en } \\
\text { cuenta el cum- } \\
\text { plimiento de las } \\
\text { competencias } \\
\text { laborales. }\end{array}$ & $\begin{array}{l}\text { Incurrir en gas- } \\
\text { tos por efectuar } \\
\text { pagos que no co- } \\
\text { rresponden con el } \\
\text { desempeño real } \\
\text { de determinados } \\
\text { trabajadores. }\end{array}$ & $\begin{array}{l}\text { Nueve trabajadores que perci- } \\
\text { ben un salario excesivo. } \\
=11047 \text { USD/año. }\end{array}$ \\
\hline \multicolumn{5}{|c|}{\begin{tabular}{l|l} 
Total = 235642 USD/año
\end{tabular}} \\
\hline
\end{tabular}

Entre los principales disfuncionamientos que se pudieron cuantificar constan: tiempos improductivos por parte de los trabajadores y la existencia de trabajadores cuya evaluación del desempeño no corresponde con su desempeño real. En costos ocultos el hotel gasta como promedio anual- mente 235642 USD/año como se muestra en la tabla 3. Para revertir esta situación, se propone diseñar los procesos de la GRH.

Con base en el estudio realizado, se rediseñaron los procesos de competencias laborales, de selección e integración del personal, de evaluación del desempeño y de capacita- 
ción y desarrollo. El proceso de competencias laborales es el más necesario por su influencia en el desempeño de los procesos claves de la GRH, fundamentalmente en la selección e integración del personal, la evaluación del desempeño y la capacitación y desarrollo, las cuales no se ejecutaban mediante un enfoque de competencias. Los disfuncionamientos identificados en estos tres últimos procesos son provocados, entre otras causas, por no gestionarse por competencias.

Otro elemento esencial del análisis de costos realizado en la investigación demuestra que diversos de los disfuncionamientos existentes en la GRH ocasionan costos ocultos, los cuales al no ser identificados no son cuantificados ni controlados; sin embargo, inciden en los resultados del centro. De ahí la necesidad de definir diversos indicadores como herramientas para hacer una medición fina y precisa de tales costos en el centro y en consecuencia tomar las medidas pertinentes para controlarlos. Para ello se propone considerar los indicadores definidos por Savall y Zardet (2016) exceptuando la rotación del personal por aplicarse ya en el hotel. Estos indicadores son:

- Ausentismo

- Accidentes e incidentes de trabajo y enfermedades profesionales

- No calidad

- Falta de productividad directa

- Fluctuación laboral

De igual forma, se diseñaron en el hotel otros indicadores asociados a los disfuncionamientos elementales identificados en la GICH:

- Porcentaje de trabajadores que reciben capacitación no acorde con su perfil de puesto.
- Porcentaje de trabajadores que no son idóneos para ocupar su cargo.

- Porcentaje de trabajadores cuya evaluación del desempeño no corresponde con su desempeño real.

- Porcentaje de funciones incumplidas por falta de motivación.

- Porcentaje de trabajadores que no son ejemplares y son reconocidos.

- Tiempos improductivos o de inactividad por parte de los trabajadores.

- Porcentaje de trabajadores indirectos en cada una de las áreas.

Además, se diseñó el mapa estratégico del hotel y se presentaron algunos de los elementos que lo componen y se fiscalizó mediante un tablero de control.

Una vez diseñados los procesos, con base en los requisitos de la Norma Cubana 3001: 2007 y los disfuncionamientos encontrados, se puede mostrar los avances alcanzados en los procesos, tales como: capacitación y desarrollo, comunicación institucional, evaluación del desempeño y selección e integración, donde todos logran $100 \%$ de cumplimiento. El proceso de desarrollo de competencias avanza hasta un $90 \%$ como se muestra en la figura 5.

Para evaluar el impacto económico de los costos ocultos se tomó en cuenta la alternativa del diseño e implementación de los procesos, con base en los requisitos de la Norma Cubana 3001: 2007, la cual ofrece una herramienta esencial para una acertada $\mathrm{GRH}$, lo que favorece a una adecuada gestión del tiempo. Estos elementos permitieron eliminar un costo total de 52904.78 USD, partiendo de los disfuncionamientos identificados, causados por no tener diseñados los procesos con base en la norma cubana. 
Figura 5. Cumplimiento de los requisitos de la NC 3000-3002:2007 después de la aplicación de las mejoras

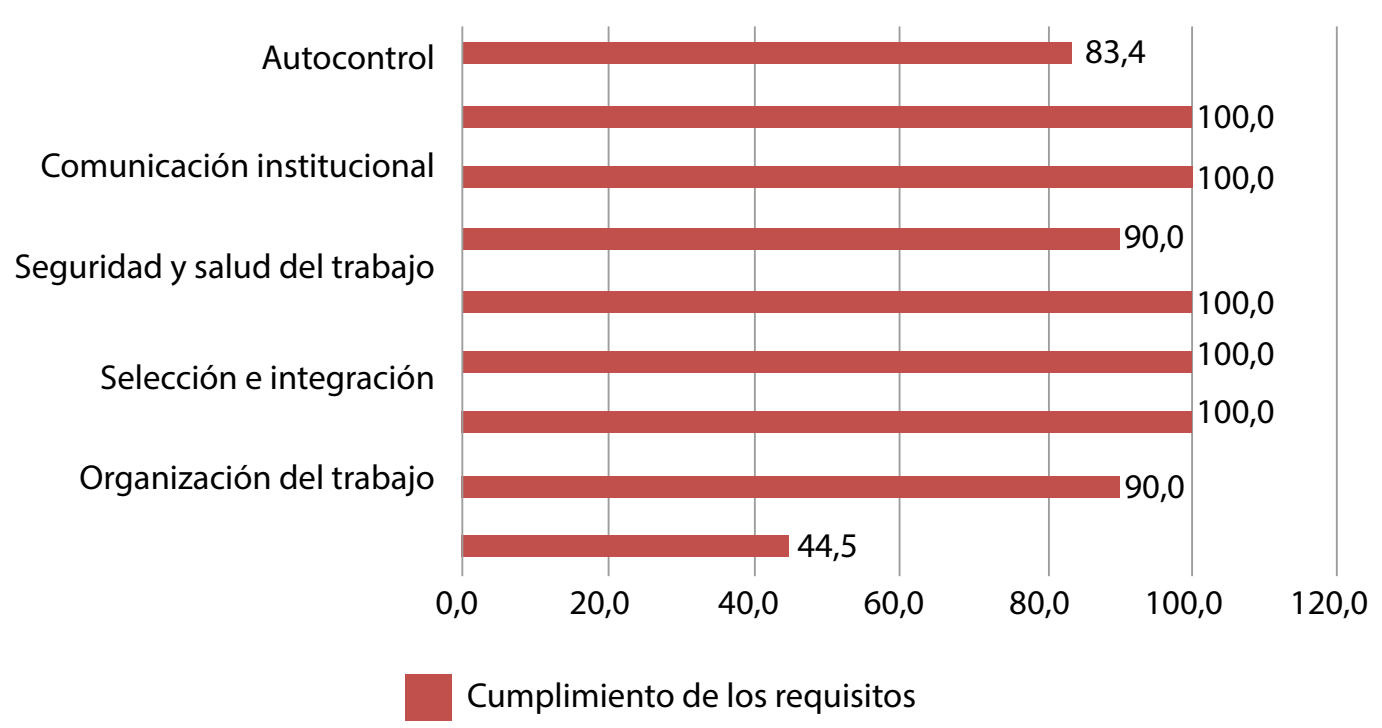

A partir de los elementos diagnosticados y las soluciones propuestas, se plantean dos alternativas:

1. No hacer nada, lo que conlleva a que la empresa genere costos ocultos anuales de aproximadamente 646 920.61USD/año.

2. Implementar los procesos de desarrollo de competencias, selección e integración, evaluación y capacitación y desarrollo, basados en los requisitos de la Norma Cubana 3001: 2007 y los disfuncionamientos detectados, lo que contribuirá a disminuir la desmotivación de los trabajadores, así como los tiempos improductivos y la existencia de trabajadores cuya evaluación del desempeño no corresponde con su desempeño real. Estos elementos permiten eliminar un costo total de 52904.78 USD/año.
Después de evaluar la relación costobeneficio, se escogió la alternativa 2 por el impacto económico que causa para el hotel.

Para la retroalimentación de la aplicación del modelo se evaluó constantemente la implementación de los procesos; no obstante, el tiempo de aplicación es de nueve meses, lo que impidió hacer un análisis integral de los procesos, ya que se recomienda que la revisión sea anual. Además, se lograron eliminar algunos disfuncionamientos existentes en el hotel, que en su mayoría están influidos por el incumplimiento de los requisitos de la Norma Cubana 3001: 2007 (ver figura 6).

El modelo permite al hotel identificar, entre los principales disfuncionamientos que se pueden cuantificar, la desmotivación, los tiempos improductivos por parte de los trabajadores, la existencia de trabajadores cuya evaluación del desempeño no corresponde con su desempeño real. 
Figura 6. Existencia de disfuncionamientos antes y después de aplicado el modelo

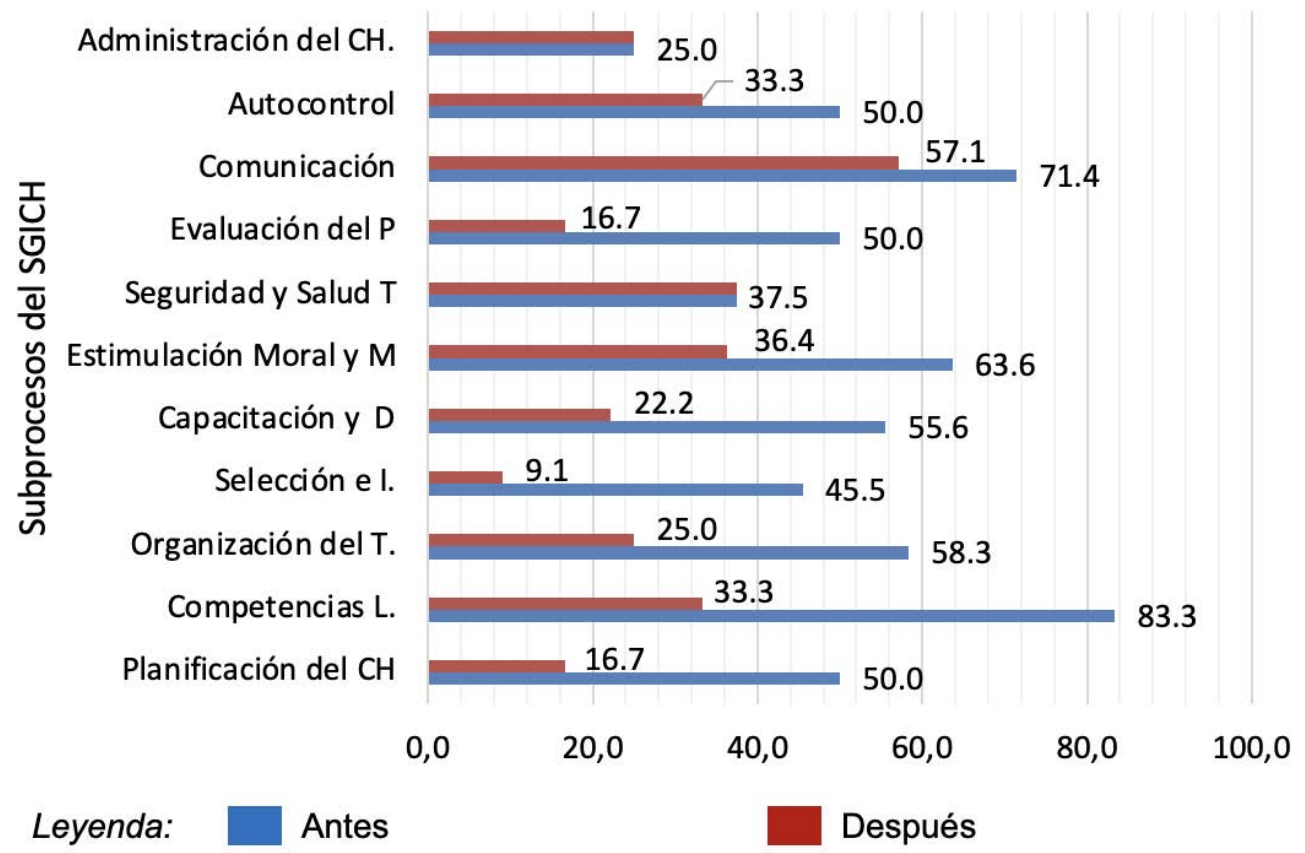

En las propuestas de mejoras se diseñaron procesos de la $\mathrm{GRH}$, en las que se puede apreciar cómo se logró llegar en muchos casos al estado deseado. Se destacan la evaluación del desempeño que de un cumplimiento inicial de 76.90 \% pasó a un $100 \%$ después de aplicar el procedimiento; y la capacitación y desarrollo que de un cumplimiento inicial de 62.5 se logró cumplir a $100 \%$ (ver figura 7).

Figura 7. Comparación del cumplimiento de los requisitos de la NC 3000-3002: 2007, antes y después

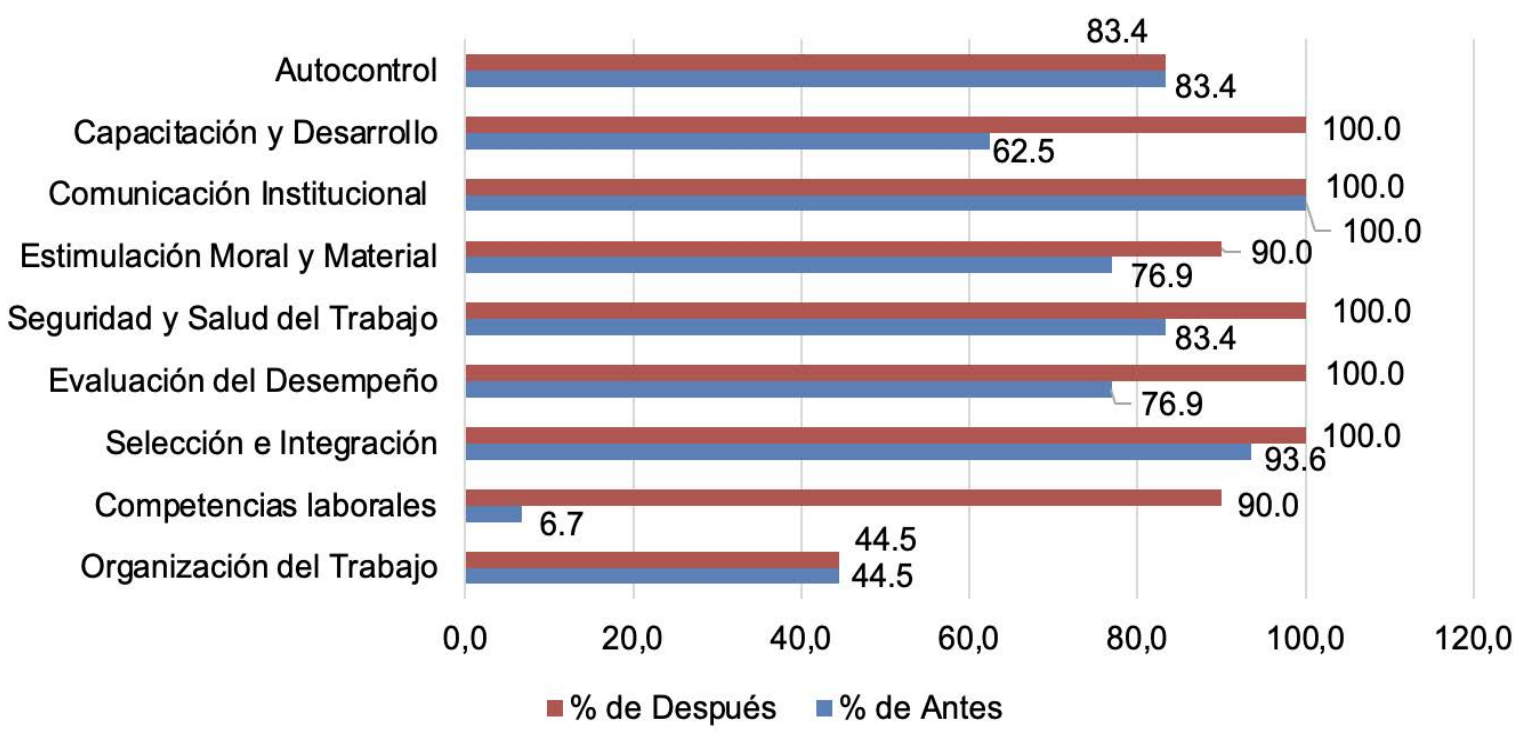




\section{Conclusiones}

Se concluye que la identificación de disfuncionamientos que generan costos ocultos en la GRH constituye una piedra angular en la gestión socioeconómica, la cual está ausente en los actuales modelos de recursos humanos.

El modelo de gestión de costos ocultos para la mejora continua de los recursos humanos se distingue por integrar aspectos sociales, así como económicos, y dentro de estos los costos ocultos que se generan a partir de disfuncionamientos existentes en la GRH con base en la satisfacción del cliente.

Con la aplicación del modelo de gestión de costos ocultos para la mejora continua de los recursos humanos se logró cuantificar los costos ocultos, los cuales ascendían a 235642 USD/año. Además, se elevó el cumplimiento de los requisitos de la Norma Cubana 3002, 2007, del hotel en el proceso de competencias laborales, el cual presentaba problemas de un porcentaje bajo con un $6.7 \%$ a un $90 \%$.

\section{Referencias}

Abad Alfonso, A., Frías Jiménez, R., \& García de León, N. (2016). Hacia un modelo conceptual de intangibles para instalaciones hoteleras. Retos Turísticos, 15(2).

Alagaraja, M., \& Githens, R.P. (2016). Capacity and capability building for national HRD: A multilevel conceptual framework. Human Resource Development Review, 15(1), 77-100. https://doi.org/10.1177/153448431562 3908

Alles, M. (2006). Dirección estratégica de recursos humanos: Gestión por competencias. Granica S.A.

Bampoky, B. (2012). Les dysfonctionnements révélateurs de coûts cachés dans les entreprises sénégalaises: quelles perspectives pour la maximisation de la valeur? Revue Congolaise de Gestion, 1-2(15-16), 127166. https://doi.org/10.3917/rcg.015.0127
Bassey, B.E., \& Tapang, A.T. (2012). Capitalized human resources cost and its influence on corporate productivity: A study of selected companies in Nigeria. International Journal of Financial Research, 3(2), 48-59. https://bit.ly/3BfXv22https://bit. ly/3BfXv22

Beer, M., Walton, R.E., Spector, B., Lawrence, P.R., \& Mills, D.Q. (1985). Human resource management: A general manager's perspective: Text and Cases. Free Press. https:// bit.ly/3bax1og

Bermúdez, H.L. (2014). Perspectivas contemporáneas de la administración estratégica de recursos humanos. Cuadernos de $\mathrm{Ad}$ ministración, 30(52), 94-104. https://bit. ly/3BTyv17

Blackman, A., Moscardo, G., \& Gray, D.E. (2016). Challenges for the theory and practice of business coaching. Human Resource Development Review, 15(4), 459-486. https:// doi.org/10.1177/1534484316673177

Blaga, P., \& Jozsef, B. (2014). Human resources, quality circles and innovation. Procedia Economics and Finance, 15, 14581462. https://doi.org/10.1016/S22125671(14)00611-X

Bríñez, M., González, A., \& Romero, V. (2016). Prospectiva del capital intelectual: Cuadro de mando integral como generador de valor empresarial. SAPIENTIAE: Revista de Ciencias Sociais, Humanas e Engenharias, 2(1), 65-80. https://bit.ly/3mu95CG

Chiavaneto, I. (2017). Administración de Recursos Humanos: El capital Humano de las Organizaciones. 10ma ed. McGraw-Hill

Chitescu, R. I., \& Lixandru, M. (2016). Tthe influence of the social, political [sic] and economic impact on human resources, as a determinant factor of sustainable development. Procedia Economics and Finance, 39, 820-826. https://doi.org/10.1016/ s2212-5671(16)30259-3

Cuesta Santos, A. (2012). Modelo integrado de gestión humana y del conocimiento: Una tecnología de aplicación. Revista Venezolana de Gerencia, 17(57), 86-98. https:// bit.ly/3ETecCH 
Cuesta Santos, A. (2015). Human resources management in the company: performance and sense of commitment. Review of Business Management, 17(56), 1134-1148. https://doi.org/10.7819/rbgn.v17i56.1736

Cuesta Santos, A. (2016). Compromiso y gestión humana en la empresa. Universitas Psychologica, 15(2), 287-300. https://doi. org/10.11144/Javeriana.upsy15-2.cghe

Cuesta Santos, A., \& Valencia Rodríguez, M. (2014). Indicadores de gestión humana y del conocimiento en la empresa. Ecoe Ediciones.

Cuesta Santos, A., \& Valencia Rodríguez, M. (2018). Capital humano: contexto de su gestión: Desafíos para Cuba. Ingeniería Industrial, 39(2), 135-145. https://bit.ly/3w76fqA

García, V., \& Fleitas, S. (2013). Procedimiento para el estudio de la organización del trabajo. Nueva Empresa, 9, 122-135.

Gemar-Castillo, G., Negrón-González, A. M. G., Lozano-Piedrahita, C. J. P., Guzmán-Parra, V. F. P., \& Rosado, N. (2019). Procedure for the continuous improvement of human resource management. Ingeniería e Investigación, 39(1), 53-62. https://doi. org/10.15446/ing.investig.v39n1.72402

Harper \& Lynch. (1992). Management estratégico y recursos humanos. Harper \& Lynch.

Herrera Ricardo, L., Velázquez Zaldívar, R., \& Pérez Campaña, M. (2020). El aseguramiento material y financiero en la educación superior cubana. Economía y Desarrollo, 163(1). https://bit.ly/3BJ5Uvr

Jasinskas, E., Streimikiene, D., Svagzdiene, B., \& Simanavicius, A. (2016). Impact of hotel service quality on the loyalty of customers. Economic Research-Ekonomska Istraživanja, 29(1), 559-572. https://doi.or g/10.1080/1331677X.2016.1177465

Jinhua, S. (2016). Research on the implementation of human resource cost management and performance pay in colleges. Proceedings of the 2016 International Seminar on Education Innovation and Economic Management (SEIEM 2016). https://doi. org/10.2991/seiem-16.2016.68

Kaplan, R., \& Norton, D. (2014). El cuadro de mando integral: The balanced scorecard. Gestión 2000.
King, K. G. (2016). Data analytics in human resources. Human Resource Development Review, 15(4), 487-495. https://doi. org/10.1177/1534484316675818

Mansor, M. F., Abu, N. H., Rashid, I. M. A., \& Sa'aban, S. (2017). Human resource outsourcing practicing: Does cost reduction and business strategy affect government link companies (GLCs)? Advanced Science Letters, 23(4), 3042-3045(4). https://doi. org/10.1166/asl.2017.7646

Matsumoto, R. N. (2014). Development of Servqual Model for the measurement of the service quality in the publicity company Ayuda Experto. Revista Perspectivas, 34, 181-209. https://bit.ly/3naYrzU

Mesa, G. P., Serra, R. T., \& Fleitas, S. T. (2009). Metodología para la gestión de los activos fijos intangibles visibles en una universidad. Revista Universidad y Sociedad, 10(4), 154-161. https://bit.ly/3m2FcZV

Montoya Monsalve, J. N., \& Montoya Naranjo, J. D. (2012). Grupo Semco y las prácticas de recursos humanos de alto compromiso: Una ventaja competitiva. INNOVAR: Revista de Ciencias Administrativas y Sociales, 22(46), 21-38. https://bit.ly/3BWtKDX

Morales Cartaya, A. (2009). Capital humano: Hacia un sistema de gestión en la empresa cubana. Editora Política.

Negrón-González, A. M. G., Gemar-Castillo, G. C., \& Noda, M. E. H. (2020). Identificación de costos ocultos relacionados con la gestión de competencias laborales. Ciencias Holguín, 26(1), 15-29.

Oficina Nacional de Normalización (ONN). (2007a). NC 3000:2007. Sistema para la Gestión Integrada de Capital Humano - Vocabulario. Oficina Nacional de Normalización.

Oficina Nacional de Normalización (ONN). (2007b). NC 3001:2007. Sistema para la Gestión Integrada de Capital Humano - Requisitos. Oficina Nacional de Normalización.

Oficina Nacional de Normalización (ONN). (2007c). NC 3002:2007. Sistema para la Gestión Integrada de Capital Humano - Implementación. Oficina Nacional de Normalización.

Parra-Acosta, J.F., \& Peña-González, Y.C. (2014). La teoría de los costos - desempeños 
ocultos: Una aproximación teórica. Cuadernos de Contabilidad, 15(39). https:// doi.org/10.11144/Javeriana.cc15-39.tcdo

Peralta Gómez, M.C., Santofimio, A.M., \& Segura, V. (2007). El compromiso laboral: Discursos en la organización. Psicología desde el Caribe, (19), 81-109. https://bit. ly/3aZnU9L

Pereda, S. M., \& Berrocal, F. B. (2018). Dirección y gestión de recursos humanos por competencias. Centro de Estudios Ramón Areces.

Quintanilla, I. (1991). Recursos humanos y marketing interno. Editorial Pirámide.

Savall, H. (2011). Por un trabajo más Humano. Information Age Publishing.

Savall, H., \& Zardet, V. (2016). Medición del efecto palanca de la cohesión sobre el desempeño económico, según la teoría del zócalo estratégico. Contaduría Universidad de Antioquia, 0(67), 55-82. https://bit. ly/3aZGwGL

Savall, H., \& Zardet, V. (2017). Strategic engineering of the reed: Reflections on socio- economic strategy and implementation. Information Age Publishing. https://bit. ly/3b0012l

Savall, H., \& Zardet, V. (2019). Reciclar los costos ocultos durables: La gestión socioeconómica. Método y resultados. Administración y Organizaciones, 8(16), 17-43. https:// bit.ly/3jpfVrO

Stone, D. L., \& Deadrick, D. L. (2015). Challenges and opportunities affecting the future of human resource management. Human Resource Management Review, 25(2), 139-145. https://doi.org/10.1016/j. hrmr.2015.01.003

Vardarlıer [sic], P. (2016). Strategic approach to human resources management during crisis. Procedia: Social and Behavioral Sciences, 235, 463-472. https://doi. org/10.1016/j.sbspro.2016.11.057

Werther, W. B., \& Davis, K. (2008). Administración de personal y recursos humanos: El capital de las empresas. McGraw-Hill, Interamericana. 